Nota científica

(Short communication)

\title{
SCOLYTUS SCHEVYREWI Y EUWALLACEA CA. FORNICATUS (COLEOPTERA: CURCULIONIDAE: SCOLYTINAE) EN TIJUANA, BAJA CALIFORNIA, MÉXICO
SCOLYTUS SCHEVYREWI AND EUWALLACEA CA. FORNICATUS (COLEOPTERA: CURCULIONIDAE: SCOLYTINAE) IN TIJUANA, BAJA CALIFORNIA, MEXICO

\author{
José Tulio MÉNDEZ-MONTIEL ${ }^{1}$, Rodolfo CAMPOS-BOLAÑOS ${ }^{1}$, THOMAS H. ATKINSON ${ }^{2}$, SiLVIA \\ EDITH GARCÍA-DÍAZ ${ }^{1 *}$
}

\author{
${ }^{1}$ División de Ciencias Forestales, Universidad Autónoma Chapingo, km. 38.5 Carretera México-Texcoco, C.P. 56230, Chapingo, \\ Edo.de México.<jmendezm@taurus.chapingo.mx>; <rcampos@correo.chapingo.mx>; <edith65@gmail.com> \\ ${ }^{2}$ University of Texas Insect Collection, 3001 Lake Austin Blvd., Suite 1. 314, Austin, Texas 78702, EE. UU. \\ $<$ thatkinson.austin@gmail.com> \\ *Autor de correspondencia: <edith65@gmail.com>; <edithgar@correo.chapingo.mx> \\ Recibido: 08/10/2018; aceptado: 18/11/2019; publicado en línea: 05/12/2019 \\ Editor responsable: Arturo Bonet Ceballos
}

Méndez-Montiel, J. T., Campos-Bolaños, R., Atkinson, T. H., García-Díaz, S. E. (2019) Scolytus schevyrewi y Euwallacea ca. fornicatus (Coleoptera: Curculionidae: Scolytinae) en Tijuana, Baja California, México. Acta Zoológica Mexicana (nueva serie), 35, 1-6. https://doi.org/10.21829/azm.2019.3502090

RESUMEN. Se reporta la asociación del descortezador exótico Scolytus schevyrewi y el barrenador ambrosial Euwallacea ca. fornicatus, en árboles de parques, jardines y camellones de la ciudad de Tijuana, Baja California, México.

Los barrenadores ambrosiales exóticos deben ser considerados con mayor atención, dándoles la importancia que requieren dentro de un sistema de alerta temprana altamente eficiente, basado en un trampeo suficiente que ayude a tomar acciones oportunas para evitar daños en especies nativas. Los insectos descortezadores (fleófagos) y barrenadores ambrosiales (xilomicetófagos) de las subfamilia Scolytinae, pueden presentarse atacando a un hospedante en forma conjunta o por separado (Wood, 1982). En olmos (Ulmus pumila L.), se presentan ataques de los descortezadores Scolytus multistriatus (Marsham) y Scolytus schevyrewi Semenov sin la presencia de barrenadores ambrosiales (Méndez \& Equihua, 1999; Campos et al., 2015). En este trabajo se encontró al barrenador ambrosial Euwallacea ca. fornicatus atacando olmos acompañado del descortezador S. schevyrewi. Euwallacea ca. fornicatus tiene un amplio rango de hospedantes, en Asia y Estados Unidos, siendo el aguacate (Persea americana Mill.), uno de los de mayor importancia económica en México (SENASICA, 2015; Carrillo et al., 2016).

Scolytus schevyrewi, barrenador del olmo, fue detectado por primera vez en el centro de México por Campos et al. (2015), sobre U. pumila. Rabaglia et al. (2006), por primera vez reportaron a Euwallacea fornicatus (Eichhof) en el sur de California y el sur de Florida, en Estados Unidos. Posteriormente, Stouthamer et al. (2017) con técnicas genéticas encontraron que se trata de un complejo de especies (por lo menos tres), morfológicamente indistinguibles, por lo que se han confundido con este nombre. Los autores antes citados, usaban los nombres "polyphagous short hole borer" para la especie establecida en la cuenca 
de Los Ángeles, California, y el nombre "kuroshio shot hole borer" para la especie presente más al sur en la región de San Diego, California. En el año 2016 se registró por primera vez en México una especie del complejo E. ca. fornicatus reportándola como "kuroshio shot hole borer" en Tijuana, Baja California (García et al., 2016). Equihua et al. (2016), ubicaron en el Centro Cultural de Tijuana, Baja California a Euwallacea sp., afectando varios árboles de Casuarina cunninghamiana Miq. estos últimos autores no indicaron la especie genética, pero lo más probable es que se trate de "kuroshio shot hole borer". El objetivo del presente reporte es dar a conocer los resultados encontrados durante la visita realizada los días 7 y 8 de junio del 2017 a la ciudad de Tijuana, Baja California.

Se visitó el "Parque Morelos" (Fig. 1), ubicado en la ciudad de Tijuana, Baja California $\left(32.499495^{\circ} \mathrm{N}, 116.936242^{\circ} \mathrm{O}\right)$, donde un mes antes se habían realizado trabajos de control de $E$. ca. fornicatus, se observaron y revisaron siete ejemplares de $U$. pumila muertos.

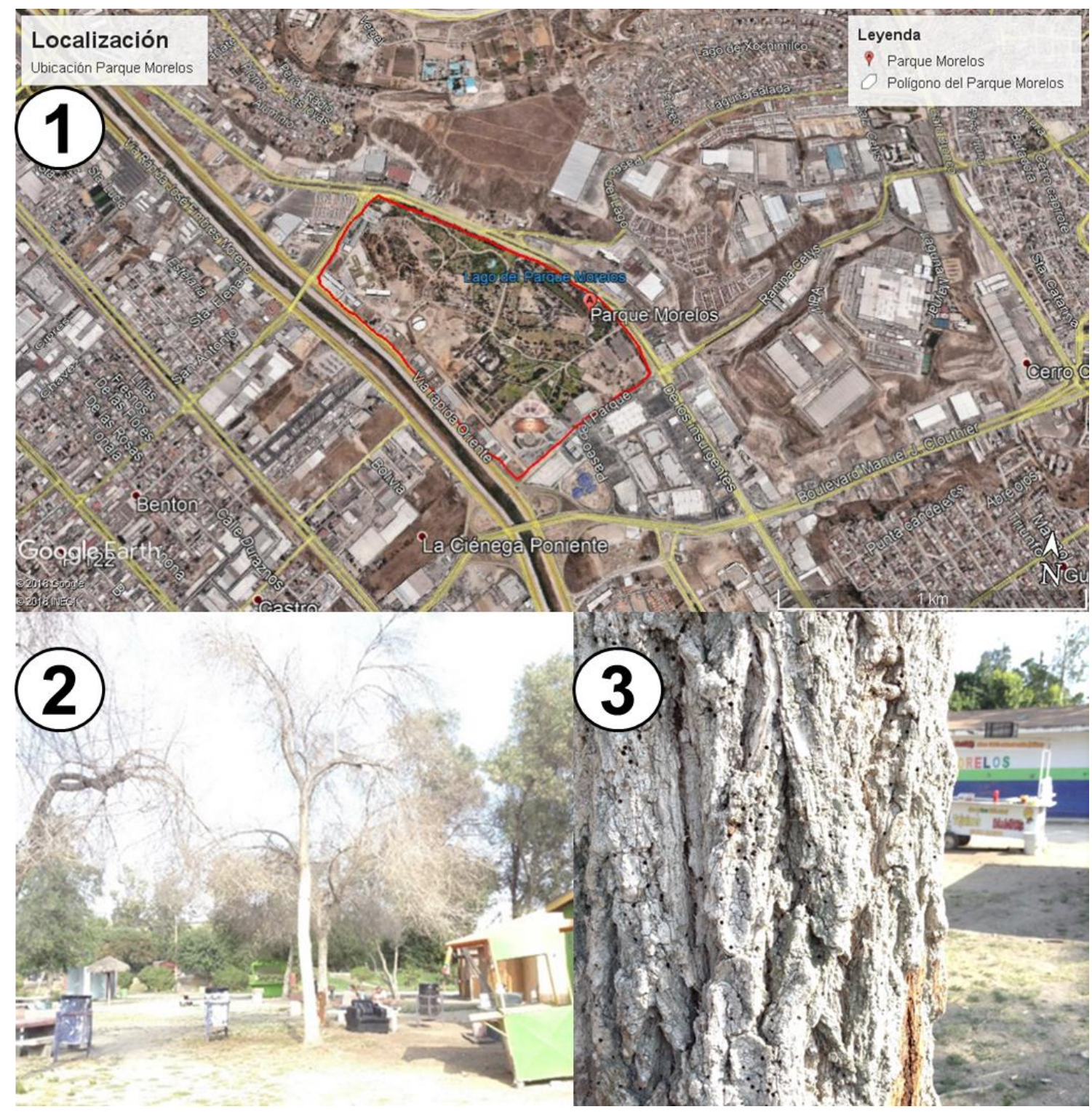

Figuras 1-3. Scolytus schevyrewi. 1) Ubicación del Parque Morelos, Tijuana, Baja California, México. 2) Grupo de olmos muertos en el Parque Morelos, por S. schevyrewi. 3) Orificios de salida del descortezador S. schevyrewi en olmo. 
Los resultados muestran que los siete árboles muertos presentaron ataques de descortezadores del género Scolytus con una gran cantidad de orificios de emergencia (Figs. 2,3) y en la madera, debajo de la corteza había evidencias de perforaciones de barrenadores ambrosiales (Figs. 4-6), al obtener una muestra de esta madera, se encontraron insectos adultos y por sus características morfológicas correspondió al género Euwallacea. Se colectaron 20 hembras de Euwallacea sp. en alcohol y cinco adultos de Scolytus sp., los cuáles se llevaron al laboratorio de Parasitología Forestal de la División de Ciencias Forestales, Universidad Autónoma Chapingo. Se utilizaron las claves de Wood (1982) para su identificación y el Dr. Thomas Atkinson, especialista a nivel mundial del grupo de Scolitinae, lo corroboró. Los insectos adultos se montaron en alfileres entomológicos para su colección y toma de fotografías, este material permanece en la colección entomológica del laboratorio antes mencionado.

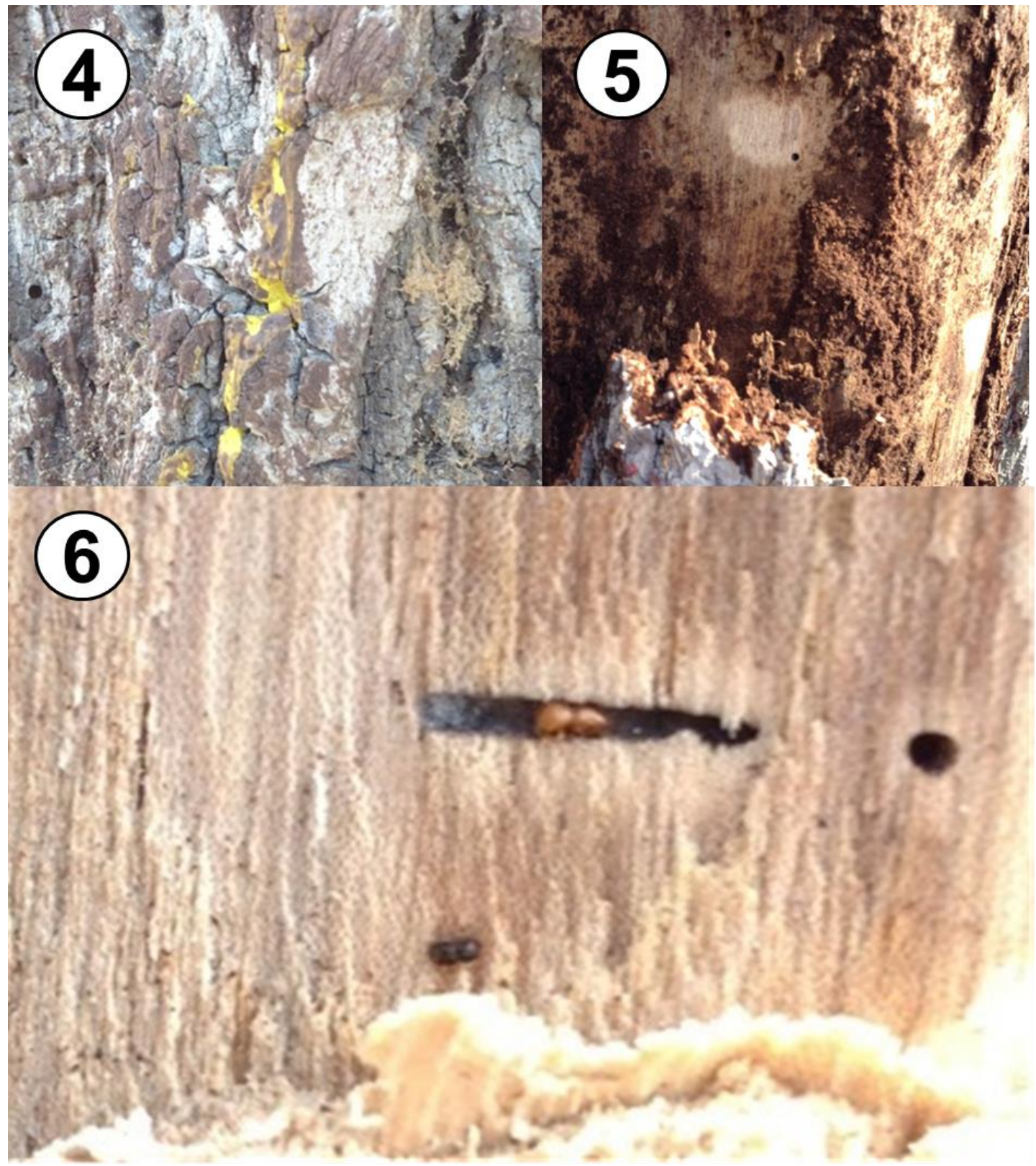

Figuras 4-6. Euwallacea ca. fornicatus. 4) Orificios de salida del descortezador y evidencias de ataque del barrenador (presencia de aserrín). 5) Orificio de entrada en la madera del barrenador. 6) Galería en la madera y dos ejemplares adultos del mismo. 
Se concluyó que los especímenes colectados son Scolytus schevyrewi (Figs. 7,8), descortezador exótico de Ulmus pumila y el barrenador ambrosial Euwallacea ca. fornicatus (Figs. 9,10). Stouthamer et al. (2017) recientemente demostraron que hay dos genotipos diferentes del complejo de Euwallacea ca. fornicatus que se han establecido en el sur de California. Por el momento no se pueden distinguir morfológicamente, pero por razones de cercanía geográfica la especie colectada en Tijuana, debe pertenece al genotipo designado como "Kuroshio shot hole borer" (Rabaglia et al., 2006; Stouthamer et al., 2017).

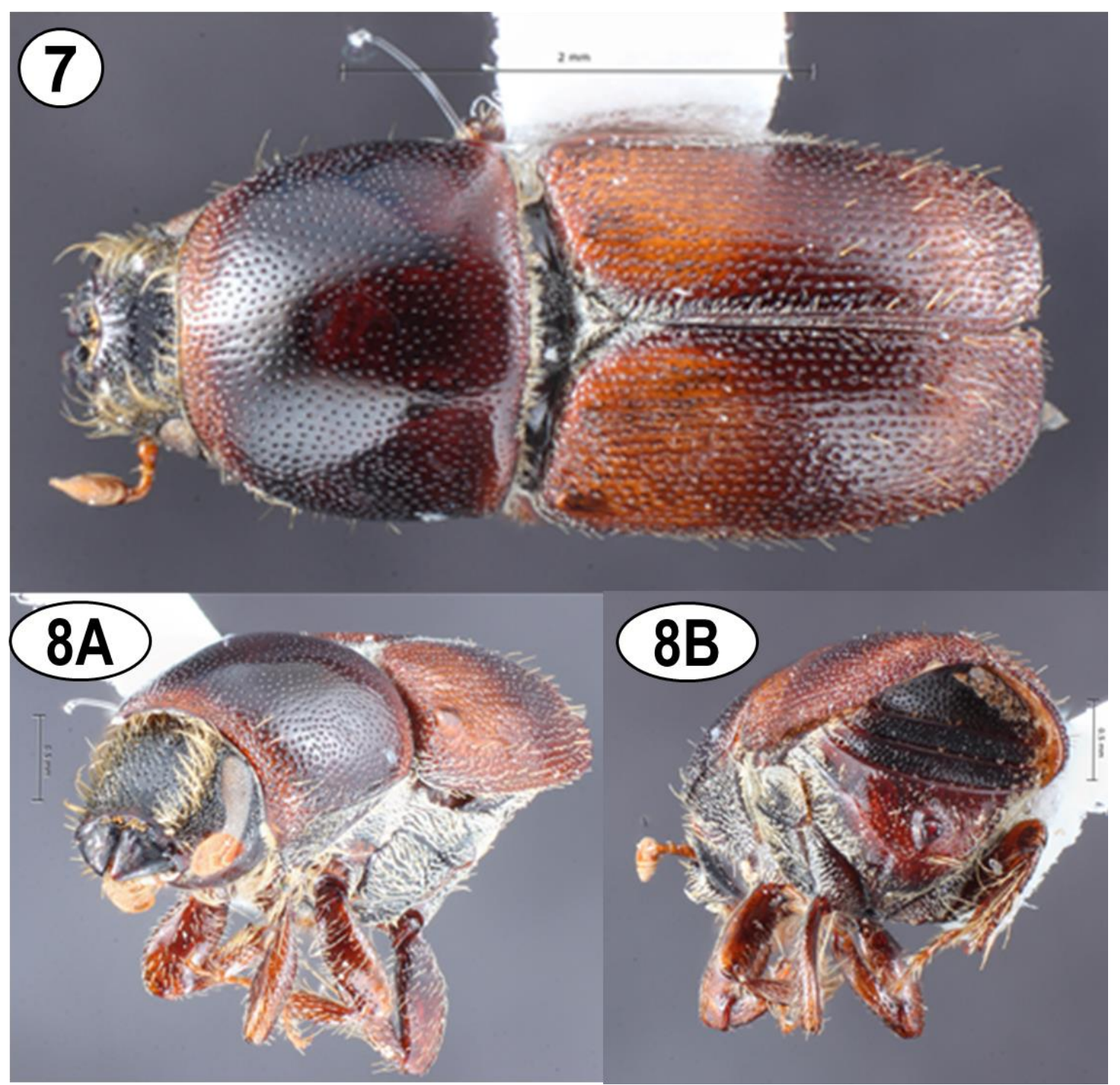

Figuras 7-8. Scolytus schevyrewi adulto. 7) Vista dorsal. 8A) Vista frontal. 8B) Vista posterior.

Este es el primer registro documentado de la asociación en $U$. pumila de $S$. schevyrewi y $E$. ca. fornicatus, lo cual resulta relevante por el hecho de que olmos muertos por el descortezador, posteriormente son atacados por E. ca. fornicatus, considerado este último de alta importancia y por ende no debe pasar desapercibido y realizar un programa de manejo integrado de barrenadores ambrosiales exóticos.

Se recomendó a los responsables de manejo del Parque Morelos, realizar la corta inmediata de los olmos muertos y el quemado de la madera inmediatamente después de ser cortados y troceados con la finalidad de eliminar focos de infestación. 
Se debe realizar un diagnóstico fitosanitario en el arbolado (incluyendo los olmos) de los parques, jardines y camellones de la ciudad de Tijuana, porque es bien conocido que E. ca. fornicatus, está en la ciudad y es necesario, saber con mayor precisión donde se encuentra y realizar urgentemente las medidas de sanidad pertinentes con la finalidad de evitar su dispersión. Por lo que en su momento esto fue informado a las autoridades de la Comisión Nacional Forestal (CONAFOR).

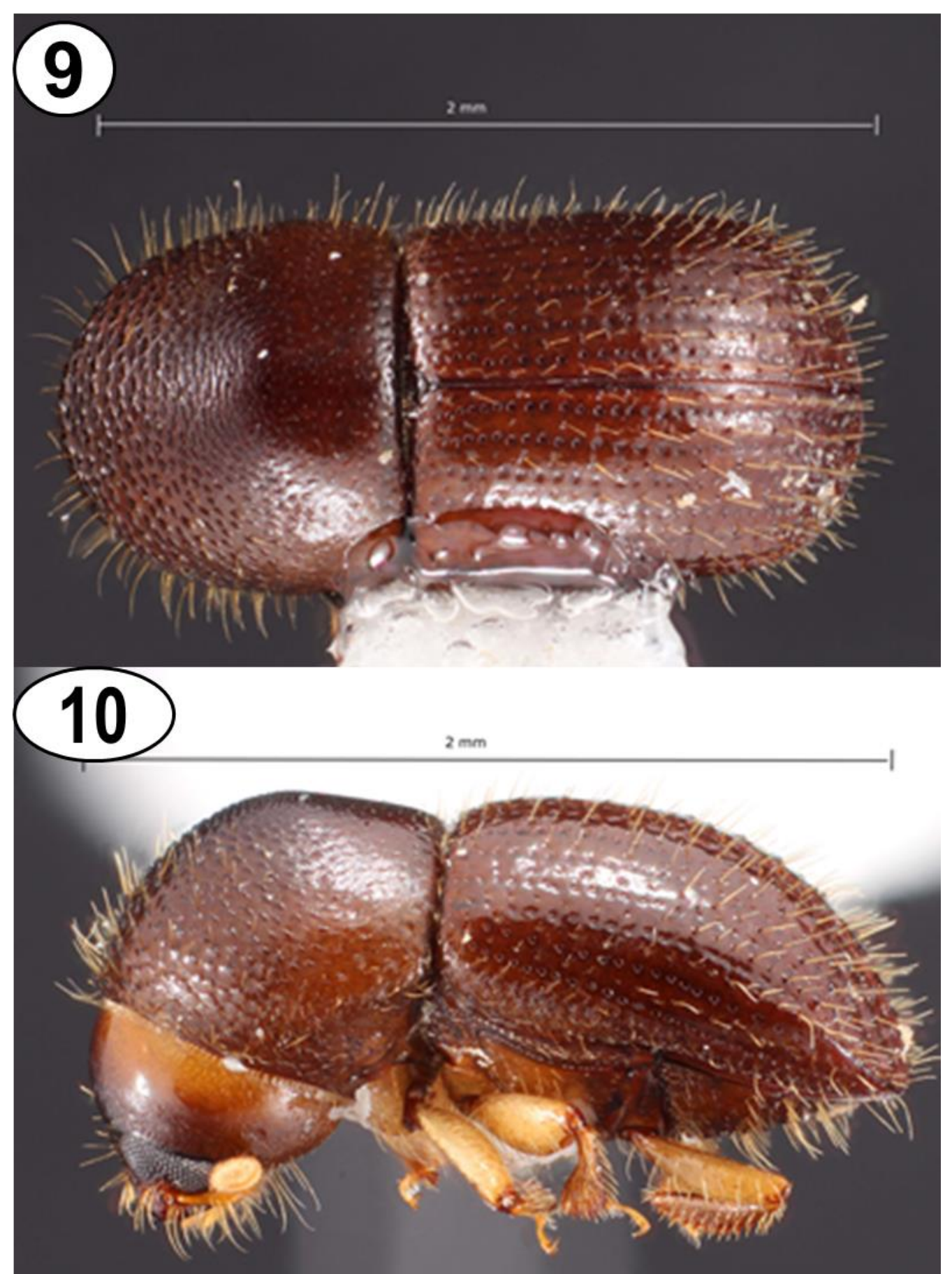

Figuras 9-10. Euwallacea ca. fornicatus adulto macho. 9) Vista dorsal. 10) Vista lateral. 


\section{LITERATURA CITADA}

Campos, R., Atkinson, T., Cibrián, D., Méndez, T. (2015) Primer registro de Scolytus schevyrewi Semenov 1902 (Coleoptera: Curculionidae: Scolytinae) en México. Acta Zoológica Mexicana (nueva serie), 31 (1), 146-148.

Carrillo, D., Cruz, L. F., Kendra, P., Narváez, T., Montgomery, W., Monterroso, A., Cooperband, M. F. (2016) Distribution, pest status and fungal associates of Euwallacea nr. fornicatus in Florida avocado groves. Insects, 7 (4), 55. https://doi.org/10.3390/insects7040055

Equihua, A., Estrada, E., Trujillo, J., García, C., López, A., Quezada, A., Ruíz, I., González, R., Gómez, J. M., Montiel, C. J., Alvares, C. B., Laureano, A., Plascencia, G. (2016) Nueva asociación entre Euwallacea sp. (Coleoptera: Curculionidae: Scolytidae) y Casuarina cunninghamiana Miq. (Casuarinaceae) en Tijuana, Baja California Norte, México. Folia Entomologica Méxicana (nueva serie), 2 (1), 20-21.

García, A. C., Trujillo, A. F., López, B. J., González, G. R., Carrillo, D., Cruz, L. F., Ruiz, G. I., Quezada, S. A., Acevedo, R. N. (2016) First report of Euwallacea nr. fornicatus (Coleoptera: Curculionidae) in Mexico. Florida Entomologist, 99 (3), 555-556.

Méndez, M. J. T., Equihua, M. A. (1999) Presencia e importancia de Scolytus multistriatus (Marsham), descortezador del olmo en Aguascalientes, Aguascalientes, México. Acta Zoológica Mexicana (nueva serie), 76, 1-15. https://doi.org/10.21829/azm.1999.76761696

SENASICA. Servicio Nacional de Sanidad, Inocuidad y Calidad Agroalimentaria-Dirección General de Sanidad Vegetal-Programa de Vigilancia Epidemiológica Fitosanitaria (2015) Polyphagous shot hole borer (Euwallacea sp.). México, D.F. Ficha Técnica No. 62. 25 p.

Rabaglia, R. J., Dole, S. A., Cognato, A. I. (2006) Review of American Xyleborina (Coleoptera: Curculionidae: Scolytinae) occurring north of Mexico, with an illustrated key. Annals of the Entomological Society of America, 99 (6), 1034-1056.

Stouthamer, R., Rugman-Jones, P., Thu, P. Q., Eskalen, A., Thibault, T., Hulcr, J., Wang, L. J., Jordal, B. H., Chen, C. Y., Cooperband, M., Lin, C. S., Kamata, N., Lu, S. S., Masuya, H., Mendel, Z., Rabaglia, R., Sanguansub, S., Shih, H. H., Sittichaya, W., Zong, S. (2017) Tracing the origin of a cryptic invader: phylogeography of the Euwallacea fornicatus (Coleoptera: Curculionidae: Scolytinae) species complex. Agricultural and Forest Entomology, 19, 366-375. https://doi.org/10.1111/afe.12215

Wood, S. L. (1982) The bark and ambrosia beetles of North and Central America (Coleoptera: Scolytidae), a taxonomic monograph. Great Basin naturalist memoirs, 6, 1-1356. 